\title{
Laboratory Validation Study of Using Time-Lapse Oscillatory Pumping Test for Leakage Detection in Geological Repositories
}

\author{
Alexander Y. Sun* \\ Jiemin $\mathrm{Lu}$ \\ Akand Islam \\ *Corresponding Author: alex.sun@beg.utexas.edu \\ Bureau of Economic Geology \\ Jackson School of Geosciences \\ The University of Texas at Austin \\ Austin, TX
}




\begin{abstract}
Geologic repositories are extensively used for disposing byproducts in mineral and energy industries. The safety and reliability of these repositories are a primary concern to environmental regulators and the public. Time-lapse oscillatory pumping test (OPT) has been introduced recently as a pressure-based technique for detecting potential leakage in geologic repositories. By routinely conducting OPT at a number of pulsing frequencies, an operator may identify the potential repository anomalies in the frequency domain, alleviating the ambiguity caused by reservoir noise and improving the signal-to-noise ratio. Building on previous theoretical and field studies, this work performed a series of laboratory experiments to validate the concept of timelapse OPT using a custom made, stainless steel tank under relatively high pressures. The experimental configuration simulates a miniature geologic storage repository consisting of three layers (i.e., injection zone, caprock, and above-zone aquifer). Results show that leakage in the injection zone led to deviations in the power spectrum of observed pressure data, and the amplitude of which also increases with decreasing pulsing frequencies. The experimental results are further analyzed by developing a 3D flow model, using which the model parameters are estimated through frequency domain inversion.
\end{abstract}

Keywords: oscillatory pumping test; sandbox experiments; leakage detection; geologic repositories; carbon sequestration 


\section{Introduction}

Geologic repositories have been extensively used in the mining and energy industries to dispose process byproducts (e.g., waste water). In recent years, geologic carbon sequestration (GCS), which involves injection of carbon dioxide $\left(\mathrm{CO}_{2}\right)$ into saline aquifers and depleted oil and gas reservoirs, is being actively pursued as an intermediate option for reducing greenhouse gas emission to the atmosphere (International Energy Agency, 2013). While some high-risk geologic repositories are equipped with engineered barrier systems (e.g., nuclear waste repositories), most geologic repositories rely on a site's natural geological and geochemical systems to retain the injectants in place. Thus, the long-term integrity of natural barriers in these geologic repositories becomes a primary concern to regulators, who often mandate that routine environmental monitoring be performed, not only during operations, but also long after site closure, for mass accounting and potential leakage detection. Although a large number of subsurface monitoring technologies are available today, only a few of them are suitable for long-term, deep subsurface, operational monitoring. Some commonly adopted selection criteria include the sensitivity of a device or technology to the process being monitored, its spatial and temporal coverage and resolution, and its reliability and durability. In addition, the cost of monitoring technologies is an important factor to consider when designing long-term monitoring networks. In GCS, pressurebased sensing often appears at the top of the list after applying the aforementioned selection criteria (Jenkins et al., 2015).

Like many geophysical methods, pressure-based monitoring technologies may be classified as either passive or active technologies, depending on whether active sources (e.g., pumping/extraction wells) are used to stimulate the reservoir to generate different pressure response patterns. Active monitoring is implicitly used in so-called hydraulic tomography in 
hydrogeology, which typically involves conducting a series of pumping or slug tests at different locations or depth intervals (sources) while recording pressure responses simultaneously from an array of pressure transducers (receivers). Over the last two decades, a large number of numerical, sandbox, and shallow aquifer experiments have demonstrated that inversion of hydraulic tomography data can resolve the spatial distributions of aquifer flow properties (hydraulic conductivity, and specific yield or storage) better than conventional characterization techniques (Berg and Illman, 2011; Brauchler et al., 2007; Cardiff and Barrash, 2011; Illman et al., 2007; Jiménez et al., 2015; Mao et al., 2013; Zhu and Yeh, 2005).

In addition to varying pressure stimulation locations, recent studies also looked into the merits of modulating stimulation rates during aquifer tests (Cardiff et al., 2013a; Guiltinan and Becker, 2015; Rabinovich et al., 2015; Soueid Ahmed et al., 2016; Zhou et al., 2016) and petroleum reservoir characterization (Ahn and Horne, 2010; Fokker and Verga, 2011), reviving the concept of oscillatory pumping test (OPT) or harmonic pulse testing that was introduced as early as in 1970s (Kuo, 1972) but had only been studied intermittently over the years (Black and Kipp, 1981; Hollaender et al., 2002; Lavenue and Marsily, 2001; Rasmussen et al., 2003). During OPT, the injection or production rate is formed as sinusoidal or square waves and pressure responses are observed at monitoring wells. By propagating such coded pressure signals through a formation, OPT possesses a number of potential advantages over the conventional constant-rate pumping tests (Cardiff et al., 2013a). Specifically, at the so-called steady-periodic condition, which may take only a few cycles to reach, the signal amplitude and phase become relatively constant. Pressure data are analyzed in the frequency domain using amplitude and phase information corresponding to known pulsing frequencies, thus reducing the dimension of observations, improving efficiency of inversion, and mitigating the impact of noise at non- 
pulsing frequencies that is caused by, for example, reservoir production activities. Zhou et al. (2016) demonstrated the OPT-based hydraulic tomography using a 2D sandbox that was subjected to constant-head boundaries on both sides. Oscillatory pumping was enabled by using a custom-built hydraulic piston controlled by an electric motor with attached flywheel. They showed that OPT-based, hydraulic tomography effectively identified the sandbox layer structures.

Recently, OPT was proposed as a time-lapse, active monitoring method for leakage detection in GCS repositories (Sun et al., 2014; Sun et al., 2015). Leakage fluxes, when detectable, affect the monitored pressure signals. Such pressure perturbations are often difficult to differentiate from the background noise by using the traditional time-domain analysis. Thus, at GCS sites, operators have resorted to monitoring pressure anomalies in formations above the injection zone that are less affected by reservoir operations; such monitoring formations are often referred to as the above-zone monitoring interval (AZMI) in the literature (Hovorka et al., 2013). Routine OPTs may provide a complementary approach to above-zone monitoring. One of the main findings from theoretical and numerical studies conducted by Sun et al. (2015) is that leakage causes discernable departures in the amplitude and phase of pressure signals compared to the no-leak case, and longer pulsing periods tend to favor the detection of leakage. Subsequent field experiments carried out by the same research group at a $\mathrm{CO}_{2}$ enhanced-oil-recovery field in Mississippi, USA, further supported the feasibility of using the time-lapse OPT as a leakage detection technique; nevertheless, the field experiments were limited by field access time and some unforeseen field conditions that shortened the length of usable data (Sun et al., 2016).

The main purpose of this laboratory study is to design and perform a series of intermediate-scale tank experiments to complement existing studies, as well as to improve the 
understanding of time-lapse OPT procedure. In the following, Section 2 describes the experimental setup, data analysis, and modeling procedure, Section 3 presents experiments results, followed by discussion and conclusions.

\section{Methods}

\subsection{Experimental setup}

A stainless steel (SST) tank was custom made to model a mini 3D reservoir system for this study. The tank body consists of a cylinder (inner diameter $1 \mathrm{~m}$, height $47 \mathrm{~cm}$, rolled using 0.95cm-thick, SA-240 304 SST plate) and an inverted elliptical head (height $30 \mathrm{~cm}$, made using $0.95 \mathrm{~cm}$-thick, $304 \mathrm{SST}$ ) that are welded together (Figure 1a). The lid of the tank was waterjet cut from a $5.72 \mathrm{~cm}$ thick 304 SST plate, with the additional thickness designed to counter the extra stress imposed on the lid during injection (Figure 1b). The design pressure of the tank is conservatively estimated to be $200 \mathrm{psi}$ [1379 kpa]. An adjustable pressure release valve (McMaster-Carr adjustable brass relief valve with max pressure of 300psi [2068 kpa]) is installed at the bottom of the tank. The tank lid, which is removable using a fork lift, is attached to the tank body using uniformly spaced $1.27-\mathrm{cm}$ hexhead bolts, with two rubber O-rings installed in between for water tightness (Figure 1b). Ports were drilled on the lid and around the tank wall for instrumentation. The tank was thoroughly tested for water tightness before performing experiments.

Oscillatory pumping is implemented by using a hybrid piston-syringe pump (Car-May Novaspense ${ }^{\mathrm{TM}}$ Model 2-4, http://www.car-may.com/products.html, Berthoud, CO) and an arbitrary wave generator (Keysight 33210A 10MHz Function/Arb Waveform Gen., Santa Rosa, CA). The wave generator can modulate the injection rate to form sinusoidal, triangular, or square 
waves at different magnitudes and frequencies. The tank is instrumented using 4 custom configured high-resolution pressure transducers (Omega Engineering, Stamford, CT) with a range of $250 \mathrm{psi}$ [1724 kpa] and accuracy of $\pm 0.03 \%$. The pressure transducers are connected to a PC running Windows 7, via a data acquisition unit (OM-DAQ-USB-2401 data acquisition module, Omega Engineering, Stamford, CT) (Figure 2a).

We performed a series of single-phase experiments under two main configurations. In the first configuration, the entire tank was filled with uniform sand (Accusill 30/40, AGSCO Corp, IL). The capacity of the tank is around 1 ton of sand when completely filled. The main purpose of this first configuration was to test the instrumentation and experimental procedures, especially with regard to displacement of entrapped air. During (wet) packing, the sand was slowly added and each layer was compacted, after which the tank was closed and $\mathrm{CO}_{2}$ gas was injected from the bottom port of the tank to displace entrapped air bubbles. Each run typically starts from atmospheric pressure. Water is injected into the tank through oscillatory pumping until an upper pressure limit is reached, which is always kept below 150 psi [1034 kpa] to avoid pump damage. In principle, the net volume of injected water should be the same for the same total experimental time and injection rate, regardless of the pulsing frequency used. We verified this fact by keeping track of the water balance during experiments using scales. The consistency of oscillatory pumping frequency was also verified using a stop watch, which indicates that the pump is able to maintain consistent pumping cycles even under high pressures. For each experimental configuration, several runs were performed until the pressure increase became relatively linear at late times, indicating the gas phase was eliminated. Moreover, instead of releasing pressure when the tank is not used, it was found necessary to keep the tank under pressurized condition to keep the gas phase from forming (e.g., through exsolving from injected water). 
After an experimental procedure was satisfactorily established, the tank was configured into a three-layer system, consisting a permeable injection layer, a low-permeability confining layer (i.e., caprock), and a permeable, above-zone layer. Such stylized three-layer systems have often been adopted in previous theoretical and numerical investigations for studying leakage from GCS repositories (Cihan et al., 2011; Nordbotten et al., 2004; Sun et al., 2015; Sun and Nicot, 2012). However, few studies have attempted to create such pressurized, layered systems in a laboratory setting.

Fabrication of the confining layer took several iterations. In the beginning, we used a thin layer of clay (bentonite) as the confining layer. Clay materials are commonly used in unconfined or low-pressure confined sandbox experiments to simulate flow barriers or confining beds. In our test, however, the clay confining layer quickly yielded after only a couple of runs, as suggested by the above-zone pressure transducers. Inspection after opening the tank lid also revealed several dark grey points, suggesting that the bentonite was flushed to the top through cracks.

Eventually, we formed the confining layer by covering a thin clay layer using a $0.635 \mathrm{~cm}-$ thick aluminum divider that is caulked to the tank wall (Figure 2b). The thin clay layer was supported by another thin layer of fine sand (\#100-140) from below, which is referred to as the transition zone. Table 1 lists all layers in bottom-to-top order.

Figure 3 shows the port locations used during our three-layer experiments, with their actual coordinates listed in Table 2 . The origin of the coordinates is located at the center of the bottom plane of the cylindrical tank body. The injection port is $0.125 \mathrm{~m}$ directly above the origin. Experiments were performed using pulsing periods of 30, 60, 90, 180, and 360 s, under both noleak (baseline) and leak conditions. These ad hoc pulsing periods were chosen based on the pump's sensitivity to wave generator signals and on observed frequency responses (see Section 
3). Sinusoidal injection rates were applied in all experiments, with magnitudes varying between 0 and $40 \mathrm{ml} / \mathrm{min}$. For the leak experiments, a leakage pathway was implemented by connecting the injection layer to the above-zone layer (Port B3 to A2) using a $0.0625 \mathrm{~cm}$ [1/16 in] SST wireline that is controlled by a two-way needle valve to regulate the leak rate. All pressure transducer ports are deployed at their designated locations in the tank using $0.3175 \mathrm{~cm}$ [1/8 in] SST tubing.

\subsection{Data analyses and Modeling}

Pressure data recorded by the four pressure transducers are analyzed to remove the trend, extract the steady-periodic segments for each experiments, and then fit using Fourier series. Because initial pressures of each experiment differ, we further require that the starting and ending pressures of each pair of experiments be the same for comparison between baseline and leak experiments. The Matlab toolbox SLMtools (https://www.mathworks.com/matlabcentral/fileexchange/24443-slm-shape-language-modeling), which provides a generic curve-fitting function, is used to fit and remove the trend in raw pressure time series (see Support Information). The Matlab function fit is used to fit the trendremoved residuals to a Fourier series. A one-term sinusoid model is adopted in this study that can capture the data variations reasonably well

$$
\mathrm{D} p(t)=a_{1} \cos (w t)+b_{1} \sin (w t), \quad w=\frac{2 p}{T_{p}}
$$

in which $a_{1}$ and $b_{1}$ are fitting coefficients and $w$ is angular frequency corresponding to pulsing

period $T_{p}$. The amplitude and phase of the sinusoid are $A=\sqrt{a_{1}^{2}+b_{1}^{2}}$ and $y=\tan ^{-1}\left(a_{1} / b_{1}\right)$.

Validation of the time-lapse OPT procedure does not require a flow model per se. However, a model would be needed for parameter estimation or leak location identification. We 
demonstrate the former use in this work. A finite-element model was created to simulate tank experiments using the Darcy's flow module in multiphysics software COMSOL ${ }^{\mathrm{TM}}$ V4.4 (https://www.comsol.com). The model consists of 31,556 elements (Figure 4a). On a laptop equipped with Intel i7 $2.2 \mathrm{GHz} \mathrm{CPU}$, it takes around $89 \mathrm{~s}$ to simulate a $900 \mathrm{~s}$ experiment conducted at pulsing period of $T_{p}=30 \mathrm{~s}$ (Figure $4 \mathrm{~b}$ ). Time-domain history matching also needs to deal with ambiguity associated with data selection. Specifically, previous studies have suggested that estimation of hydraulic conductivity and specific storage is sensitive to data from different periods of pumping, for example, drawdown at early times is highly correlated with $S_{s}$ but only weakly correlated with the $K$ field between the pumping and observation locations (Mao et al., 2013; Xiang et al., 2009). Instead of time domain history matching, here a more computationally efficient phasor model is adopted (Cardiff et al., 2013a), in which a steadyperiodic groundwater flow phasor equation is solved

$$
i w S_{s} \mathrm{~F}=\tilde{\mathrm{N}} \rtimes(K \tilde{\mathrm{NF}})+Q_{s},
$$

where $K$ is hydraulic conductivity [cm/min], $S_{s}$ is specific storage [1/cm], $Q_{s}$ is injection rate $[\mathrm{ml} / \mathrm{min}], i$ is imaginary unit, and $\mathrm{F}$ is a complex variable (i.e., phasor) defined in terms of amplitude and phase given in Eq. (1), F = A exp(iy). It can be shown that $\mathrm{F}$ and Fourier coefficients are equivalent to each other (Cardiff et al., 2013b). The solution time of the phasor model using COMSOL only takes about $5 \mathrm{~s}$ on the same computer mentioned in the above. Fourier coefficients obtained from Eq.(1) are used as "observation" data to calibrate the phasor model. In this case the unknown parameters are values of $K$ and $S_{s}$ of the three layers listed in Table 1 (i.e., injection zone, transition zone, and above zone). Parameter estimation is done using the Matlab function lsqnonlin by minimizing an objective function defined as the root-mean- 
square error (RMSE) between observed and simulated Fourier coefficients that are calculated from the phasor model using each trial solution.

\section{Results and Discussion}

Raw pressure data are recorded at $0.1 \mathrm{~s}$ intervals. Figure 5 shows trend-removed, pressure residuals obtained from the injection zone pressure transducer $\mathrm{C} 1$ for pulsing periods $T_{p}=30$, 60, 90, 180, and 360 s, respectively, for both baseline and leak experiments. During all baseline experiments, the needle valve was fully closed, and during all leak experiments the needle valve was fully open (i.e., unregulated leak rate). Figure 5 suggests that all pressure residuals are reasonably fitted using a one-term Fourier series (Eq. (1)). The magnitude of pressure residuals increases with the length of pulsing periods because of longer continuous injection time in each cycle. As a result, the signal-to-noise ratio, calculated as the ratio of RMSE between signal and noise, is higher for longer pulsing periods. For example, the ratio increases from 1.9 for $T_{p}=30 \mathrm{~s}$ to 3.0 for $T_{p}=180 \mathrm{~s}$ at baseline conditions. Figure 6 compares the signals obtained from the two above-zone pressure transducers A1 and A3. The pressure signals only show apparent sinusoidal patterns under leak conditions, indicating that the confining bed was functioning as expected during baseline experiments.

It is difficult to tell the leak visually by simply examining the pressure time series obtained from baseline and leak experiments. In the next step, all pairs of sinusoids (baseline vs. leak) were transformed to frequency domain using fast Fourier transform, from which the sinusoids' amplitudes were obtained. The final results from all five pairs of experiments are shown in Figure 7, which reveals a clear deviation between baseline and leak experiments. These analyses complete one round of time-lapse OPT leak detection analysis. In practice, such 
analyses may be repeated at regular intervals to detect potential anomalies in the system. It is also suggested that the experiments be performed at multiple frequencies to confirm deviations in frequency responses. Results in Figure 7 exemplifies the main advantage of using frequencydomain analysis as opposed to time-domain analysis, namely, interference of pressure noise is reduced and, therefore, ambiguity related to time-domain signals is alleviated.

Figure 7 suggest that lower pulsing frequencies tend to reveal leak-induced deviations better, which is consistent with previous studies (Sun et al., 2016; Sun et al., 2015). Interestingly, the leak experiments all showed a positive deviation (i.e., increase in magnitude) from the baseline's amplitude. The analytical solution of Sun et al. (2015), which assumes infinite boundaries, predicts that a leak will cause amplitude attenuation from the baseline. We believe that the main cause of positive amplitude deviations observed in the current setting is due to the confined nature of the tank experiments. In the baseline experiments, the confining bed is pushed up toward the above zone to absorb some of the pressure increases induced by injection. In leak experiments, however, fluids leaked to the above zone acted to limit the upward movement of confining bed, effectively increasing pore pressure. The fact that all leak experiments showed an upward deviation in amplitudes indicates the existence of such hydromechanical coupling under closed boundary conditions. We also examined the phase shift of various baseline vs. leak experiment pairs (not shown). However, there are no consistent variations among different experiments, which agrees with observations in previous numerical experiments (Sun et al., 2015).

Parameter estimation was performed using leak experiment data according to the procedure described under Section 2.2. The final results are reported in the last two columns of Table 1 . The results are generally comparable to hydraulic conductivity $(K)$ values measured at 
similar spatial scales in the literature. For example, Schroth et al. (1996) reported a $K$ value of $8.9 \mathrm{~cm} / \mathrm{min}$ for \#30/40 sands (i.e., our injection zone) using constant-flux column experiments. Wietsma et al. (2009) measured $K$ value of \#30/40 sands using five different methods/apparatus and the results ranged from 4.6 to $8.9 \mathrm{~cm} / \mathrm{min}$. The same authors also reported the range of $K$ values of \#70 sands (i.e., our above zone) to be 0.3 to $0.8 \mathrm{~cm} / \mathrm{min}$. In their sandbox experiments, Berg and Illman (2012) measured $K$ values of F35 sand ( \#35) and F65 sand ( \#65) to be around 1.6 and $0.3 \mathrm{~cm} / \mathrm{min}$, respectively; the specific storage of sands are reported to be on the order of $10^{-5} \mathrm{~cm}^{-1}$. Our experiments were conducted at pressures much higher than those previous sandbox experiments because of the imposed closed boundary condition. The slight movement of aluminum divider during experiments, as described in the above, caused slight decrease of pore space in the above zone and increase of pore space in the zones below. The specific storage, or equivalently total compressibility, in porous flow equation consists of contributions from fluid and rock compressibilities. The rock compressibility and permeability can both decrease with increase of pressure for unconsolidated materials such as those used here (Fatt, 1958; Wu and Pruess, 2000). Thus, the estimated parameters are best viewed as temporally averaged effective parameters.

Calibration using the phasor model approach does not directly resolve the "aliasing" issue that may occur when estimating both $K$ and $S_{s}$. In other words, part of the variability in $K$ may be explained by the inverse algorithm by calibrating $S_{s}$ (Zhou et al., 2016). Nevertheless, inversion using data from multiple frequencies may significantly improve identifiability of these parameters. 


\section{Summary and Conclusions}

In summary, this study aims to perform laboratory validation of OPT as a leak detection measure, using a custom made, miniature 3D reservoir that can withstand relatively high pressures. Our experiments, conducted at a number of different pulsing frequencies, confirmed the main principle behind the time-lapse OPT for leakage detection, namely, that a detectable leak event modifies a geologic system's frequency response function and its effect can be identified on the amplitude-frequency diagram by looking for time lapse deviations. We found that low pulsing-frequency tests have better signal-to-noise ratios than high-frequency tests and are better suited for revealing leak signals. In practice, however, tradeoffs must be made by considering experimental time and signal strength. We also demonstrated the merits of performing parameter estimation in the frequency domain using OPT data, which is a more efficient alternative to the conventional time domain history matching.

\section{Acknowledgements}

The authors would like to thank Mr. Doug Hammond from Precision Design Solutions LLC. and Mr. Jonathan Hahne from Center for Electromechanics at UT Austin for their assistance during experimental design. The authors are grateful to the AE Dr. Niklas Linde for handling the manuscript and to Dr. M. Cardiff and two anonymous reviewers for their constructive comments. This work was supported by the U.S. Department of Energy, National Energy Technology Laboratory (NETL) under grant numbers DE-FE0012231 and DE-FE0026515.

\section{References}

Ahn, S. and Horne, R., 2010. Estimating permeability distributions from pressure pulse testing, SPE Annual Technical Conference and Exhibition. 
Berg, S.J. and Illman, W.A., 2011. Three-dimensional transient hydraulic tomography in a highly heterogeneous glaciofluvial aquifer-aquitard system. Water Resources Research, 47(10).

Berg, S.J. and Illman, W.A., 2012. Improved predictions of saturated and unsaturated zone drawdowns in a heterogeneous unconfined aquifer via transient hydraulic tomography: Laboratory sandbox experiments. Journal of Hydrology, 470: 172-183.

Black, J. and Kipp, K., 1981. Determination of hydrogeological parameters using sinusoidal pressure tests: a theoretical appraisal. Water Resources Research, 17(3): 686-692.

Brauchler, R. et al., 2007. An inversion strategy for hydraulic tomography: Coupling travel time and amplitude inversion. Journal of hydrology, 345(3): 184-198.

Cardiff, M., Bakhos, T., Kitanidis, P. and Barrash, W., 2013a. Aquifer heterogeneity characterization with oscillatory pumping: Sensitivity analysis and imaging potential. Water Resources Research, 49(9): 5395-5410.

Cardiff, M. and Barrash, W., 2011. 3-D transient hydraulic tomography in unconfined aquifers with fast drainage response. Water Resources Research, 47(12).

Cardiff, M., Barrash, W. and Kitanidis, P.K., 2013b. Hydraulic conductivity imaging from 3-D transient hydraulic tomography at several pumping/observation densities. Water Resources Research, 49(11): 7311-7326.

Cihan, A., Zhou, Q. and Birkholzer, J.T., 2011. Analytical solutions for pressure perturbation and fluid leakage through aquitards and wells in multilayered-aquifer systems. Water Resources Research, 47(10): W10504.

Fatt, I., 1958. Pore volume compressibilities of sandstone reservoir rocks. Journal of Petroleum Technology, 10(03): 64-66.

Fokker, P.A. and Verga, F., 2011. Application of harmonic pulse testing to water-oil displacement. Journal of Petroleum Science and Engineering, 79(3): 125-134.

Guiltinan, E. and Becker, M.W., 2015. Measuring well hydraulic connectivity in fractured bedrock using periodic slug tests. Journal of Hydrology, 521: 100-107.

Hollaender, F., Hammond, P.S. and Gringarten, A.C., 2002. Harmonic testing for continuous well and reservoir monitoring. Paper presented at the SPE Annual Technical Conference and Exhibition, San Antonio, TX, September 29-October 2, 2002. SPE Journal, 77692.

Hovorka, S.D., Meckel, T.A. and Trevino, R.H., 2013. Monitoring a large-volume injection at Cranfield, Mississippi-Project design and recommendations. International Journal of Greenhouse Gas Control, 18: 345-360.

Illman, W.A., Liu, X. and Craig, A., 2007. Steady-state hydraulic tomography in a laboratory aquifer with deterministic heterogeneity: Multi-method and multiscale validation of hydraulic conductivity tomograms. Journal of Hydrology, 341(3): 222-234.

International Energy Agency, 2013. Technology roadmap: Carbon capture and storage. , IEA, Paris, France.

Jenkins, C., Chadwick, A. and Hovorka, S.D., 2015. The state of the art in monitoring and verification - ten years on. International Journal of Greenhouse Gas Control, 40: 312349.

Jiménez, S. et al., 2015. Prediction of solute transport in a heterogeneous aquifer utilizing hydraulic conductivity and specific storage tomograms. Water Resources Research, 51(7): 5504-5520.

Kuo, C., 1972. Determination of reservoir properties from sinusoidal and multirate flow tests in one or more wells. Old SPE Journal, 12(6): 499-507. 
Lavenue, M. and Marsily, G., 2001. Three-dimensional interference test interpretation in a fractured aquifer using the pilot point inverse method. Water Resources Research, 37(11): 2659-2675.

Mao, D. et al., 2013. Joint interpretation of sequential pumping tests in unconfined aquifers. Water Resources Research, 49(4): 1782-1796.

Nordbotten, J.M., Celia, M.A. and Bachu, S., 2004. Analytical solutions for leakage rates through abandoned wells. Water Resources Research, 40(4).

Rabinovich, A. et al., 2015. Frequency dependent hydraulic properties estimated from oscillatory pumping tests in an unconfined aquifer. Journal of Hydrology, 531: 2-16.

Rasmussen, T.C., Haborak, K.G. and Young, M.H., 2003. Estimating aquifer hydraulic properties using sinusoidal pumping at the Savannah River site, South Carolina, USA. Hydrogeology Journal, 11(4): 466-482.

Schroth, M., Istok, J., Ahearn, S. and Selker, J., 1996. Characterization of Miller-similar silica sands for laboratory hydrologic studies. Soil Science Society of America Journal, 60(5): 1331-1339.

Soueid Ahmed, A., Jardani, A., Revil, A. and Dupont, J., 2016. Joint inversion of hydraulic head and self-potential data associated with harmonic pumping tests. Water Resources Research, 52(9): 6769-6791.

Sun, A.Y., Kianinejad, A., Lu, J. and Hovorka, S., 2014. A Frequency-domain Diagnosis Tool for Early Leakage Detection at Geologic Carbon Sequestration Sites. Energy Procedia, 63: 4051-4061.

Sun, A.Y., Lu, J., Freifeld, B.M., Hovorka, S.D. and Islam, A., 2016. Using pulse testing for leakage detection in carbon storage reservoirs: A field demonstration. International Journal of Greenhouse Gas Control, 46: 215-227.

Sun, A.Y., Lu, J. and Hovorka, S., 2015. A harmonic pulse testing method for leakage detection in deep subsurface storage formations. Water Resources Research, 51(6): 4263-4281.

Sun, A.Y. and Nicot, J.-P., 2012. Inversion of pressure anomaly data for detecting leakage at geologic carbon sequestration sites. Advances in Water Resources, 44: 20-29.

Wietsma, T.W., Oostrom, M., Covert, M.A., Queen, T.E. and Fayer, M.J., 2009. An automated tool for three types of saturated hydraulic conductivity laboratory measurements. Soil Science Society of America Journal, 73(2): 466-470.

Wu, Y.-S. and Pruess, K., 2000. Integral solutions for transient fluid flow through a porous medium with pressure-dependent permeability. International Journal of Rock Mechanics and Mining Sciences, 37(1): 51-61.

Xiang, J., Yeh, T.C.J., Lee, C.H., Hsu, K.C. and Wen, J.C., 2009. A simultaneous successive linear estimator and a guide for hydraulic tomography analysis. Water Resources Research, 45(2).

Zhou, Y., Lim, D., Cupola, F. and Cardiff, M., 2016. Aquifer imaging with pressure wavesEvaluation of low-impact characterization through sandbox experiments. Water Resources Research.

Zhu, J. and Yeh, T.-C.J., 2005. Characterization of aquifer heterogeneity using transient hydraulic tomography. Water Resources Research, 41(7). 
Table 1. List of layer properties

\begin{tabular}{|l|l|l|l|l|}
\hline Layer & Layer & Material & \multicolumn{2}{|l|}{ Estimated Parameters } \\
\cline { 4 - 5 } & Thickness $[\mathrm{cm}]$ & & K [cm/min] & Ss $[1 / \mathrm{cm}]$ \\
\hline Injection zone & 57 & $\# 30 / 40$ sand & 5.57 & $3 \mathrm{e}-5$ \\
\hline Transition zone & 3 & $\begin{array}{l}\text { 3100/140 } \\
\text { sand }\end{array}$ & 0.014 & $3.9 \mathrm{e}-5$ \\
\hline Confining layer & 6 & Al and clay & - & \\
\hline Above zone & 14 & \#70 sand & 0.10 & $7.9 \mathrm{e}-7$ \\
\hline
\end{tabular}

Table 2. Port locations and functions.

\begin{tabular}{|l|l|l|l|l|}
\hline Port & $\mathbf{x}(\mathbf{c m})$ & $\mathbf{y}(\mathbf{c m})$ & $\mathbf{z}(\mathbf{c m})$ & Use \\
\hline A1 & 38.97 & 22.5 & 37.5 & Pressure (above zone) \\
\hline A2 & 0 & -50 & 37.5 & Leak outlet \\
\hline A3 & -21.65 & 12.50 & 37.5 & Pressure (above zone) \\
\hline B3 & -35.36 & -35.36 & 25 & Leak inlet \\
\hline C1 & 0 & 45 & 12.5 & Pressure (injection zone) \\
\hline C2 & 0 & 0 & 12.5 & Pressure (injection zone) \\
\hline C3 & 0 & 0 & 12.5 & Injector \\
\hline
\end{tabular}




\section{Figure Captions}

1. Design of the stainless steel tank used for the experiment: (a) side view and (b) top view.

2. (a) Experimental system, in which oscillatory pumping is enabled using a hybrid pistonsyringe pump and a wave generator; (b) aluminum plate is used as the confining bed for the three-layer miniature reservoir system.

3. Layout of ports, where horizons A, B, and C are shown on Figure 1a. Filled circles are pressure transducer ports and open circles correspond to leak path ports. See Table 2 for their coordinates.

4. OPT results obtained at $\mathrm{C} 1$ (a)-(e): baseline pressure residuals obtained at pulsing periods of $30,60,90,180$, and $360 \mathrm{~s}$; (f)-(j): leak experiments pressure residuals obtained for the same pulsing periods. Data (dots) recorded at $0.1 \mathrm{~s}$, and are fitted to 1term Fourier series model (solid line).

5. OPT results obtained at $\mathrm{C} 1$ (a)-(e): baseline pressure residuals obtained at pulsing periods of $30,60,90,180$, and $360 \mathrm{~s}$; (f)-(j): leak experiments pressure residuals obtained for the same pulsing periods. Data (dots) recorded at $0.1 \mathrm{~s}$, and are fitted to 1 term Fourier series model (solid line).

6. OPT pressure residuals obtained from above-zone pressure sensors A1 (top row) and A3 (bottom row) under baseline $(\mathrm{a}, \mathrm{c})$ and leak $(\mathrm{b}, \mathrm{d})$ condition, where solid lines correspond to models fitted using one-term Fourier series.

7. Amplitude vs. pulsing frequency diagram for all experiments. Pulsing periods are labeled on the plot. 
(a)

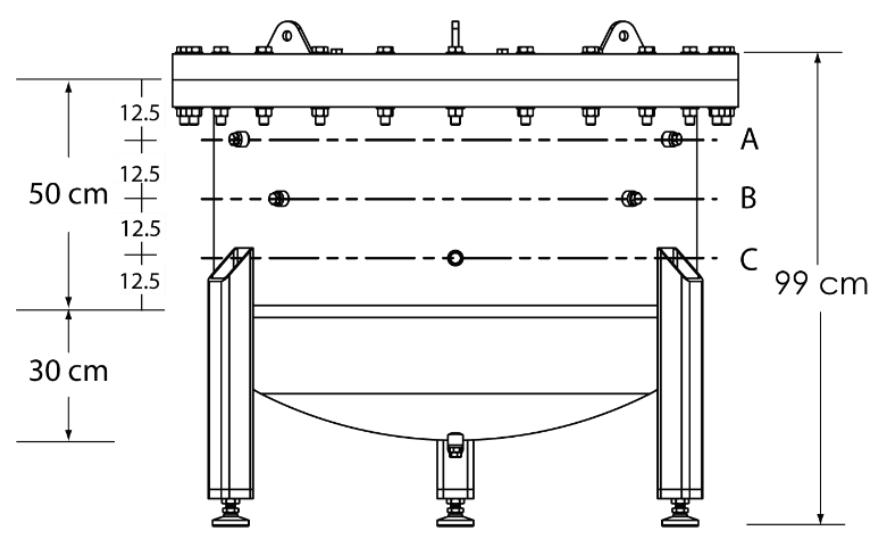

(b)

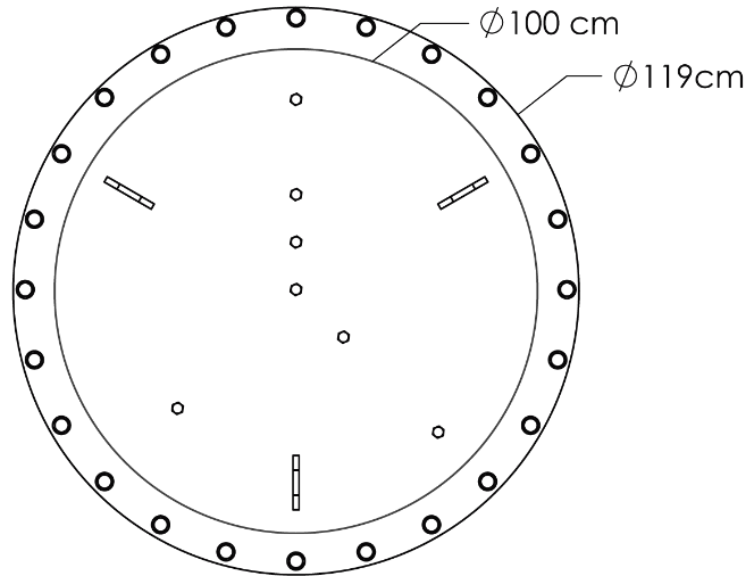

Figure 1. Design of the stainless steel tank used for the experiment: (a) side view and (b) top view. 
(a)

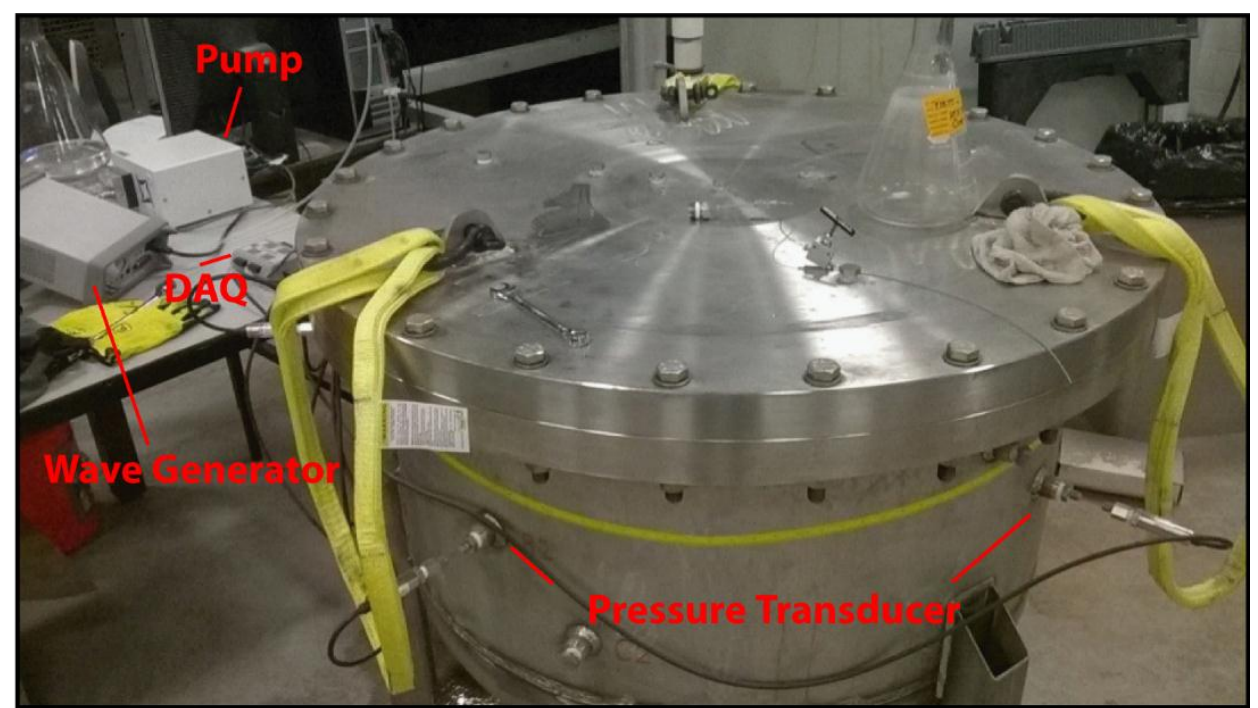

(b)

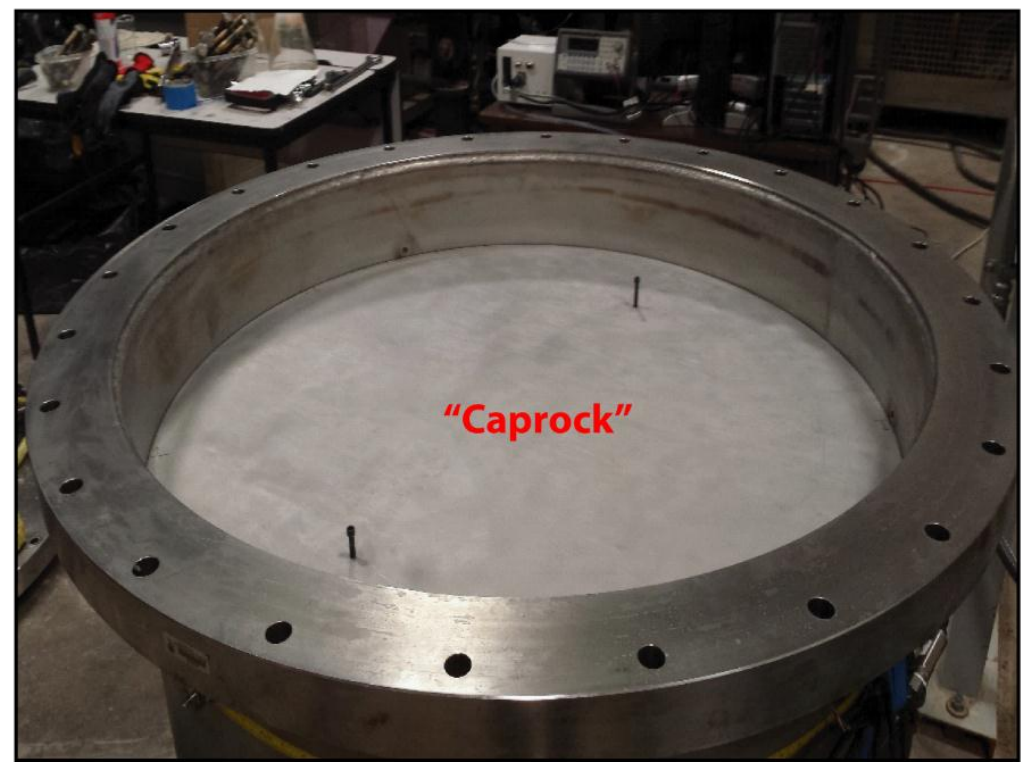

Figure 2. (a) Experimental assembly, in which oscillatory pumping is enabled using a hybrid piston-syringe pump and a wave generator, and pressure transducers are connected via a data acquisition unit (DAQ) to the desktop computer; (b) aluminum divider is used as the confining bed in the three-layer miniature reservoir system. 


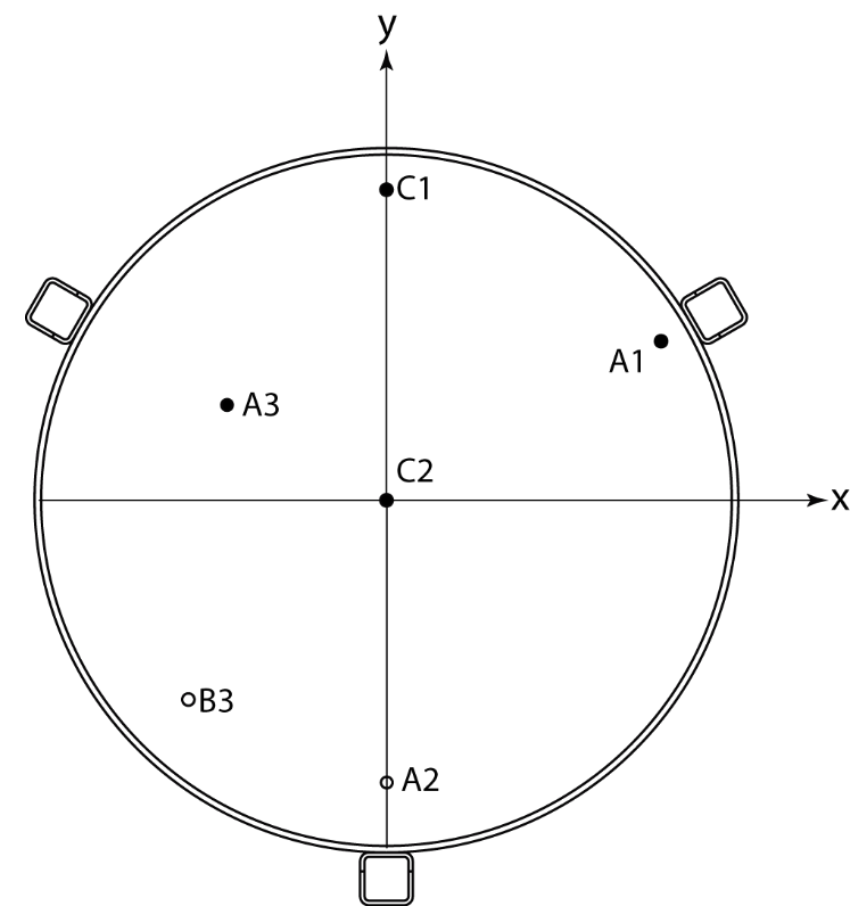

Figure 3. Layout of ports, where horizons A, B, and C are shown on Figure 1a. Filled circles are pressure transducer ports and open circles correspond to leak path ports. See Table 2 for their coordinates. 
(a)

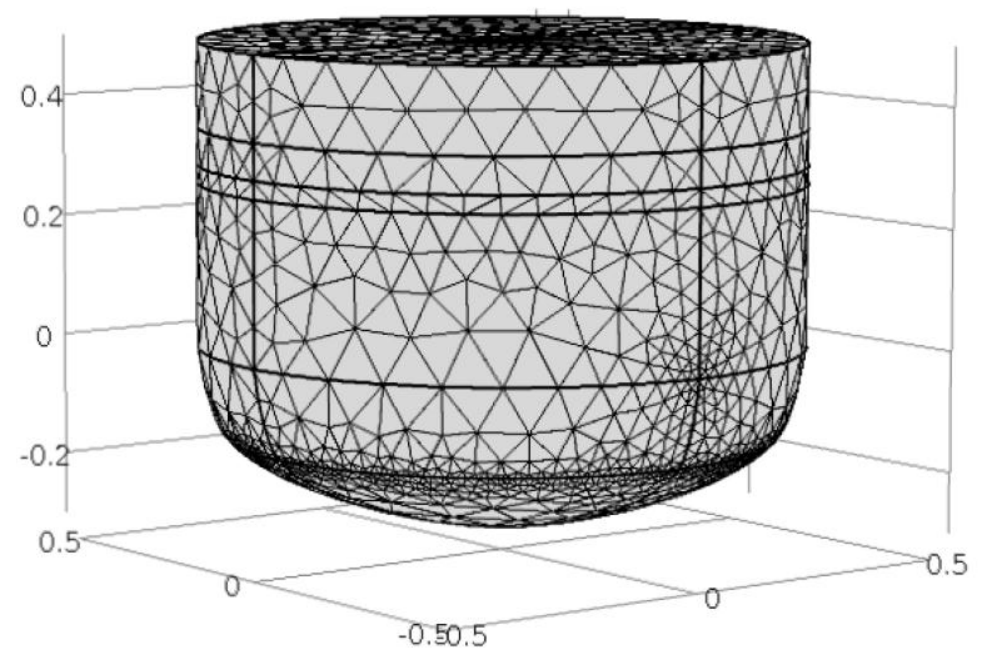

(b)

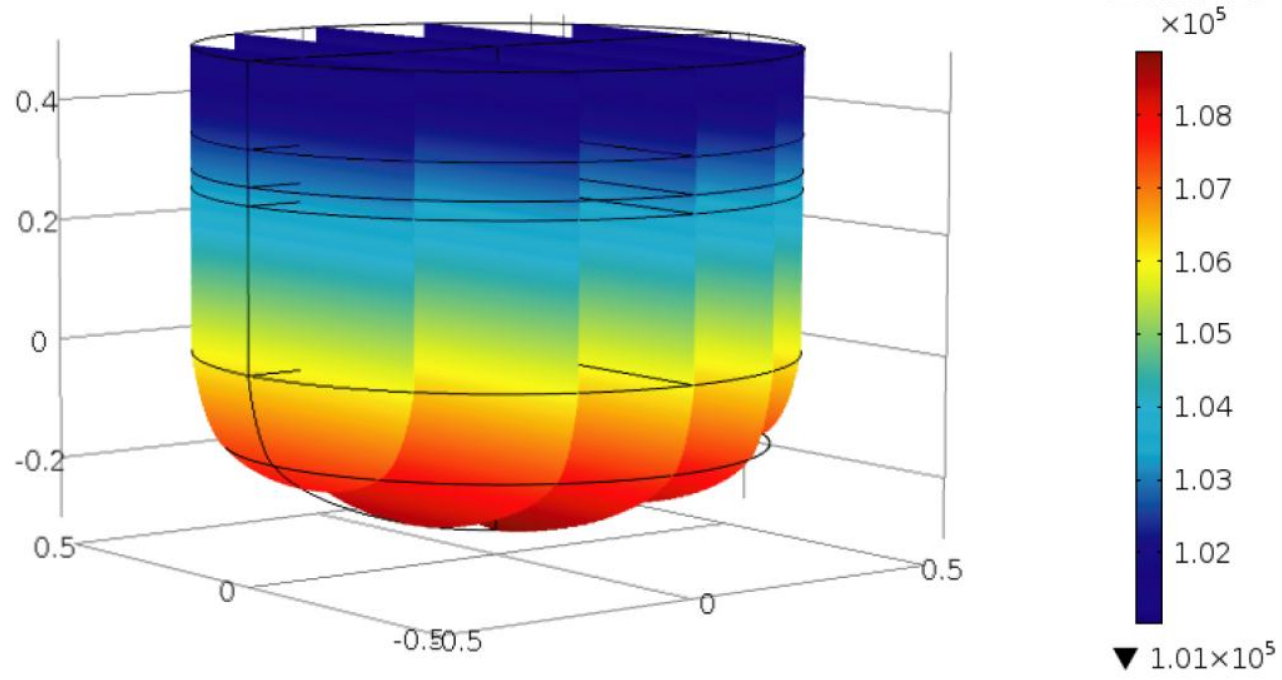

Figure 4. Finite-element model used to simulate the tank experiments: (a) the mesh has 31,556 elements; (b) pressure surface simulated for a $T_{p}=30$ s experiment. 


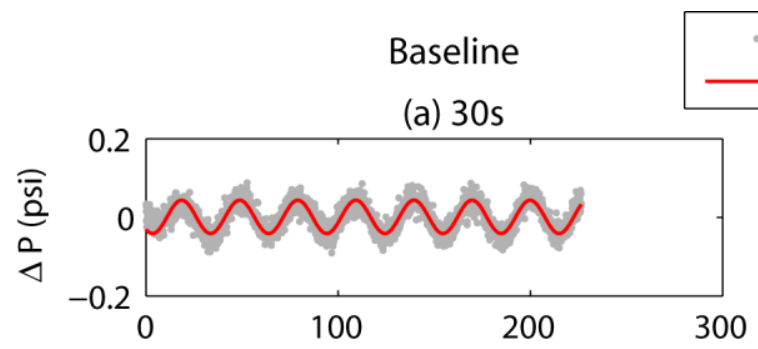

(b) $60 \mathrm{~s}$

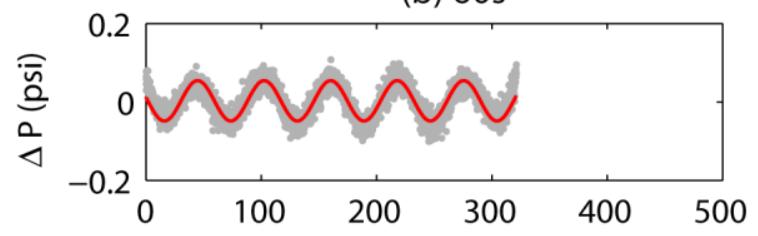

data
fitted

Leak
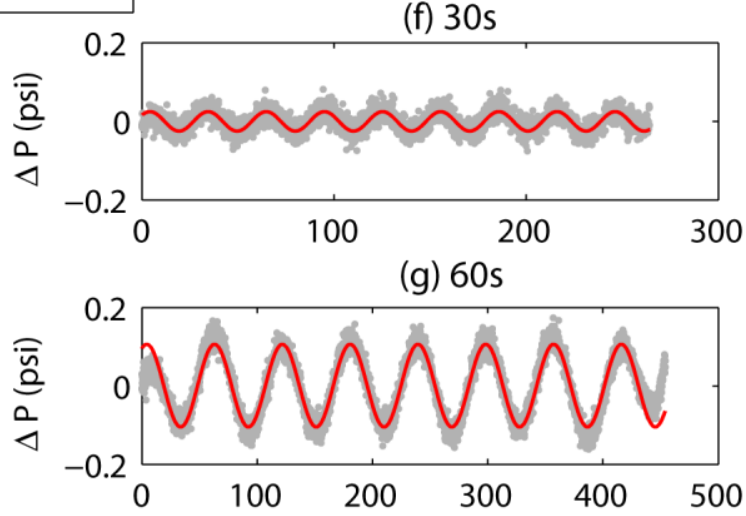

(c) $90 \mathrm{~s}$
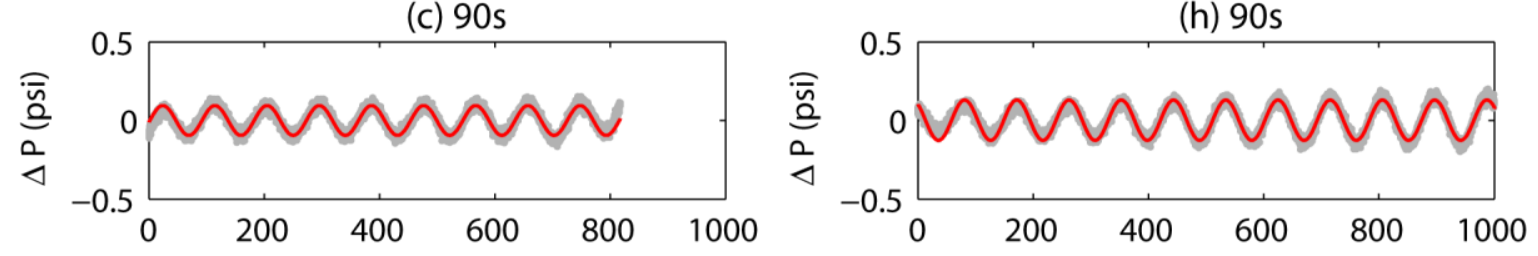

(d) $180 \mathrm{~s}$

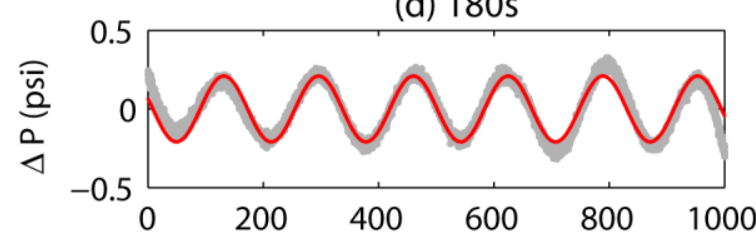

(i) $180 \mathrm{~s}$

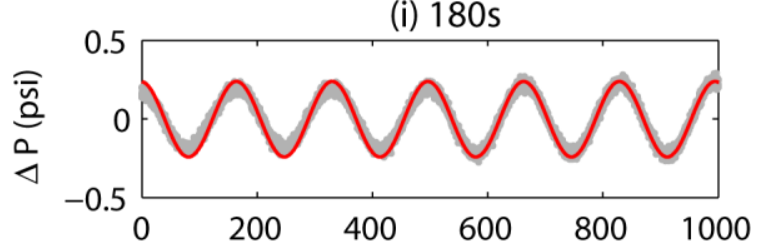

(e) $360 \mathrm{~s}$
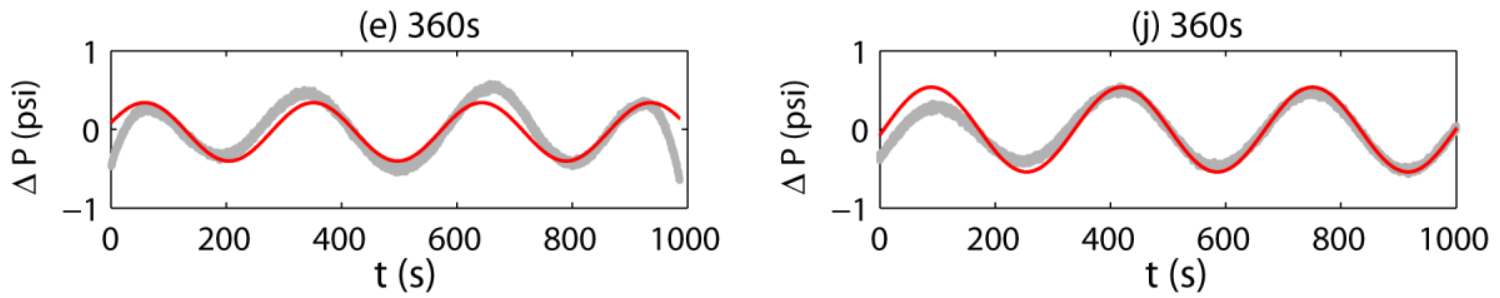

Figure 5. OPT results obtained at C1 (a)-(e): baseline pressure residuals obtained at pulsing periods $T_{p}$ of $30,60,90,180$, and 360s; (f)-(j): leak experiments pressure residuals obtained for the same pulsing periods. Data (dots) recorded at $0.1 \mathrm{~s}$, and are fitted to 1-term Fourier series model (solid line). 
A1
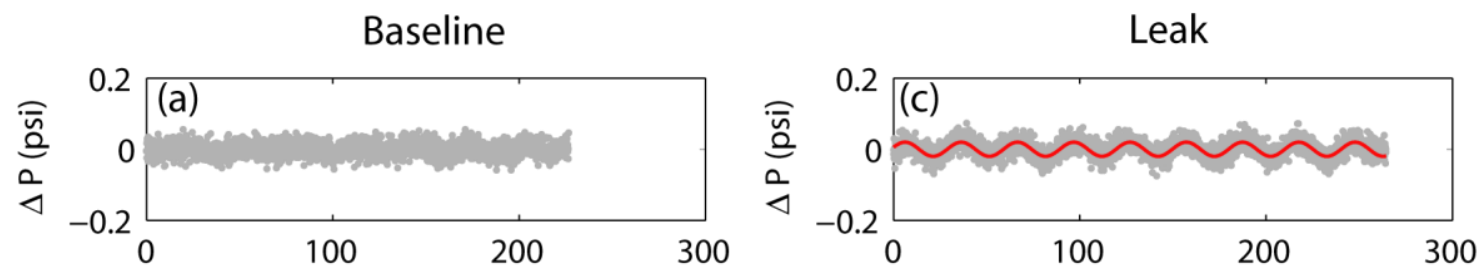

A3
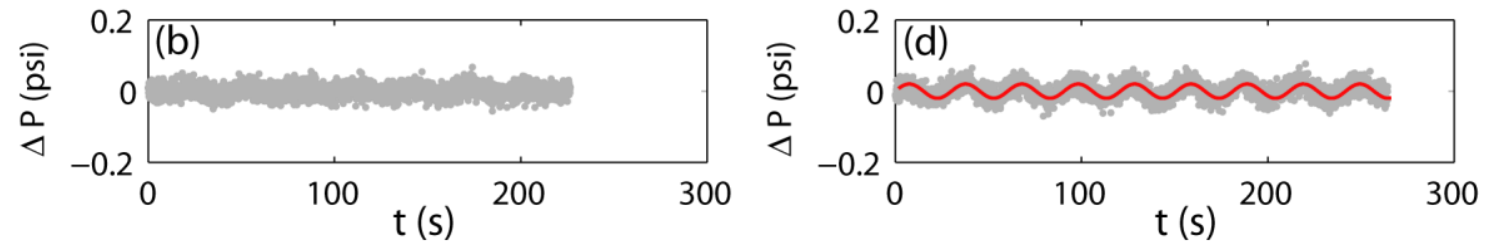

Figure 6. OPT pressure residuals obtained from above-zone pressure sensors A1 (top row) and A3 (bottom row) under baseline $(\mathrm{a}, \mathrm{c})$ and leak $(\mathrm{b}, \mathrm{d})$ condition, where solid lines correspond to models fitted using one-term Fourier series. 


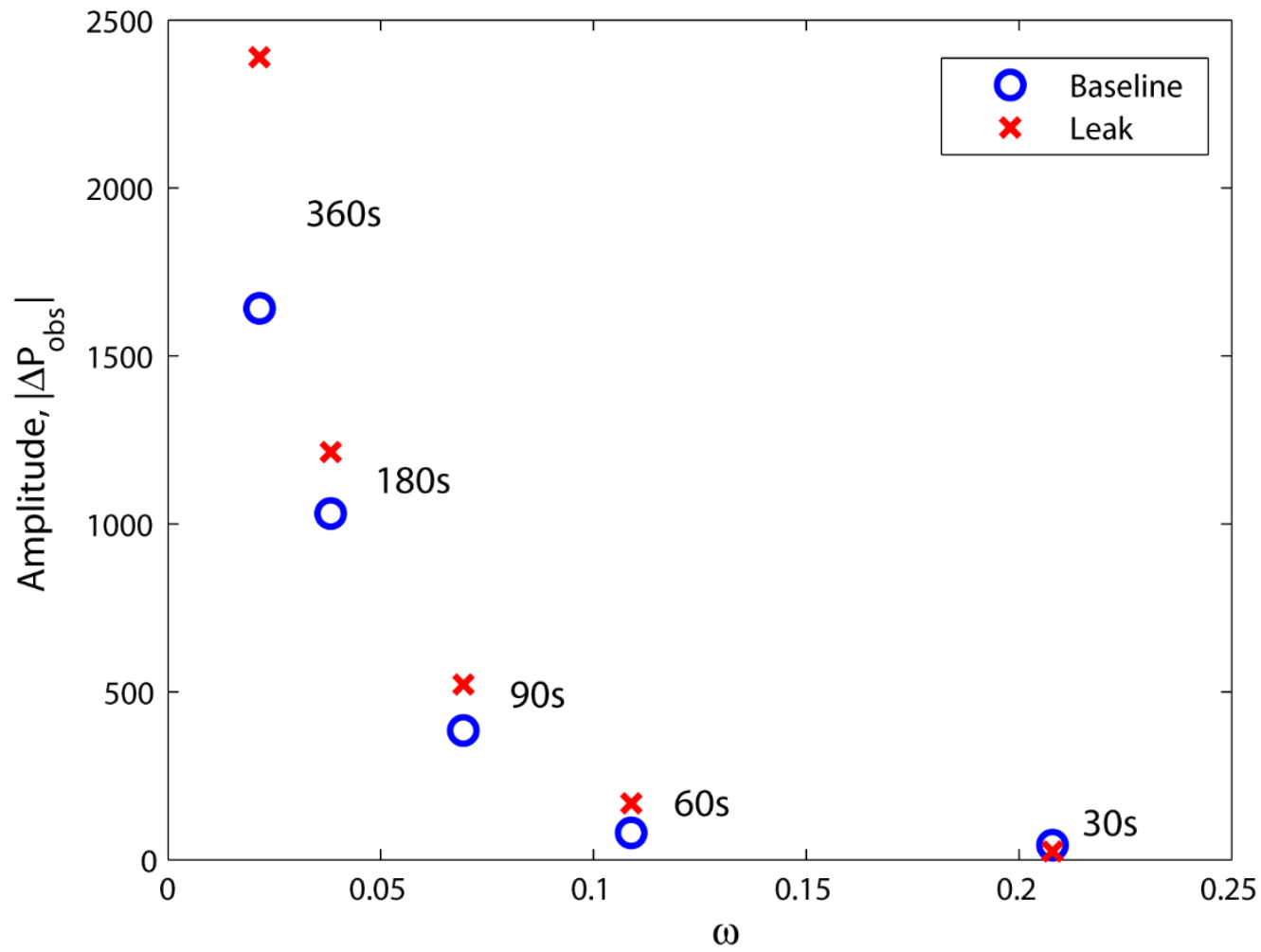

Figure 7. Amplitude vs. pulsing frequency diagram for all experiments. Pulsing periods are labeled on the plot. 